\title{
Do all associations lead to lower levels of ethnocentrism? A two-year longitudinal test of the selection and adaptation model.
}

\section{Political Behavior, 35, accepted}

(C) Springer Journals, SSCI Impact factor 1.412.

\begin{abstract}
Within the literature on the relation between membership in voluntary associations and tolerance, three theoretical perspectives can be distinguished. The socialization perspective assumes that the interaction within association leads to higher levels of tolerance; the self-selection perspective suggests that tolerant actors are more likely to join associations; while the selection and adaption model predicts that the group culture within an association will have a specific effect on members' attitudes. We use a two-year panel study among Belgian adolescents to ascertain the empirical merits of these three approaches. Results show that not all associations have an effect on the reduction of prejudice, but that this effect is limited to specific associations. A structural equation model furthermore suggests that socialization effects are significantly stronger than self-selection effects. As such, this analysis of panel data lends support to the selection and adaptation model.
\end{abstract}

Key words: voluntary associations, ethnocentrism, panel data, Belgium, socialization, selection and adaptation model 


\section{S U M M A R Y}

\section{Introduction}

Traditionally, it has been assumed that membership of voluntary associations is associated with the development of a more civic-oriented value pattern and more tolerant attitudes. Famously, Tocqueville considered associations to be a learning school of democracy as he claimed that the interaction with other members led to the enlargement of one's social perspective. The idea that voluntary associations play an important role in consolidating a democratic civic culture was again brought to attention by the Putnam (1993) volume on civic traditions in modern Italy. The subsequent research literature, however, failed to deliver conclusive evidence about the validity of this bold claim. Some studies indeed demonstrate that members of voluntary associations, on average, have significantly higher levels of generalized trust and that they are less prejudiced toward minority groups. Howard and Leah (2008) have shown that this positive relation occurs across a wide range of societies. In other studies, however, the relation between membership and civic attitudes proves to be nonsignificant (Claibourn \& Martin, 2000; Nannestad, 2008). Furthermore, even if we do observe a significant correlation, this does not inform us yet about the causal relation between both phenomena. The relation might as well be the result of endogeneity, i.e., the fact that actors with civic-minded attitudes self-select into voluntary associations (Newton, 1997). Freitag, Grießhaber and Traunmuller (2009), e.g., show for a Swiss sample that trust levels are more strongly determined by individual level characteristics than by membership of voluntary associations. More specifically, in this article, we focus on just one possible attitudinal outcome of voluntary associational involvement: the reduction of prejudice and ethnocentrism. We do so because it can be assumed that tolerance has become an important element of a democratic political culture in an ever more diverse society (Sullivan \& Transue, 1999; Hooghe, 2007). Indeed, quite some of the most recent research on social capital has focused on the relationship between diversity and social capital (Arneil, 2006; Hooghe, Reeskens, Stolle \& Trappers, 2009; Putnam, 2007). We can safely assume, therefore, that the antagonistic feelings that are present in ethnocentric prejudice run counter to the effort to strengthen social cohesion in society.

Efforts to disentangle the relationship between membership and attitudes also have to take into account the possibility that apparently not all associations are related in the same manner to these attitudes. Previous research has established quite clearly that not all kinds of 
voluntary associations have the same attitudinal impact (Paxton, 2007; van der Meer \& van Ingen, 2009; van Deth, 2010). If our goal is to establish a reliable causal mechanism, it is important that we are able to account for these observed differences between associations. If we assume that the interaction with other members is the main cause of the socialization effects, as is predicted by the Tocquevillian approach, these differences are hard to explain as interaction most likely occurs in all sorts of associations. One of the most promising explanations for this pattern is the 'selection and adaption' mechanism, based on the assumption that actors self-select into specific associations, and that they subsequently adapt to the prevailing attitudes within that interaction context (Hooghe, 2003). The evidence that is presented in favor of this mechanism, however, is thus far purely cross-sectional, and this kind of material is insufficient to be considered as a good test for the validity of this claim.

In this article, we present the results of a longitudinal study on participation in voluntary associations, that allows us to track the development of tolerant attitudes, both among members and non-members over time. Although longitudinal data by themselves do not prove causality, they are obviously better equipped to address a research question on socialization effects than the cross-sectional data that dominate this line of research, as panel data allow to control for prior attitudes. If one observes differences according to the type of association, this lends support to the central claim of the selection and adaption model. We first review the literature on the relation between membership of voluntary associations and tolerant attitudes. Subsequently we provide more information about data and methods, before presenting the results of the analysis. We close with some observations about the theoretical relevance of our findings.

\section{(...)}

\section{Data and Methods}

Testing the selection and adaption model requires panel data, as this allows us to distinguish between self-selection and socialization effects that emerge over a period of time. Data from the Belgian Political Panel Survey (BPPS 2006-2008) are ideally suited for this purpose. These data constitute a two-wave panel study among a sample of 16 and 18-year old respondents. In 2006, a representative school-based survey was conducted among 6,330 sixteen-year-olds in Belgium. The fact that this survey was conducted within a school context led to a high response rate, and an analysis of the survey responses confirms that the sample is 
representative for region, school type, gender and educational track. Based on written questionnaires completed by the respondents in 112 schools, the study focused on social and political attitudes and contained questions about civic participation. To obtain a national random sample, all schools included in the survey were selected through a stratified sample, based on the location and type of the school. In each school, a minimum of 50 students were selected, representative of the tracks offered in that school. The response rate was 66 percent for the schools, and, within the schools, 99 percent for the pupils.

In 2008, the same respondents were contacted again for a second wave, this time at the age of eighteen. While most of the initial respondents were still at the same school, alternative strategies had to be developed to reach those who had left or changed schools. Of the initial 112 schools, 109 participated again in the survey in 2008. In these schools, the same classes were re-surveyed, which allowed us to re-interview almost 3,000 students. The other students were contacted through a mail survey. In total, 4,235 pupils (or 67 percent) of the initial panel were re-surveyed. The attrition rate is in line with what can be expected for this kind of panel study: as the first wave of the panel survey was conducted in the class context, quite some pupils might have had the mistaken idea that participation was compulsory, thus inflating the initial response rate. The second wave was again representative with regard to region, school type, gender and educational track ${ }^{1}$. Given these considerations, this dataset is ideally suited to test our hypotheses. It provides access to a total sample of 4,235 panel respondents, who were interviewed both in 2006 (average age 16) and in 2008 (average age 18).

Two caveats are to observed when using this dataset. First, this is a single-country data gathering effort, that is representative for late adolescents in Belgium, a small (11 million inhabitants) founding member of the European Union. As such, there is no reason to assume that any findings from this study might be generalized toward other countries. It has to be observed, however, that both with regard to associational membership, as with regard to attitudes like ethnocentrism and trust, Belgium tends to occupy a middle position in Europe. Analyses of the European Social Survey repeatedly have shown that Belgium has lower trust and participation levels than the Scandinavian countries, but the observed levels are clearly higher than in the Southern or Eastern European countries (Davidov, Schmidt \& Schwartz, 2008). As such, there is no apparent reason to believe that Belgium would offer a highly exceptional case. A second specific element is that this panel study was conducted among adolescents and we cannot automatically assume that any findings this study might deliver can also be generalized toward an adult population. The available literature on the effects of primary socialization allows us to assume that socialization effects will be stronger among 
adolescents than among adults (McFarland \& Thomas, 2006; Pacheco, 2008). Focusing on adolescents, therefore, maximizes the likelihood that any meaningful relations will be detected, that can provide the material for the analysis. While of course among adults too, political learning processes and political socialization do occur, most likely these processes are less intensive and therefore they would require longer observation periods (Sigel, 1989). The main dependent variable in the analysis is ethnocentrism, i.e., a feeling of hostility toward outgroup members that, in the European context, is mainly directed toward immigrant groups within the population (Meuleman, Davidov \& Billiet, 2009; Sides \& Citrin, 2007). There are three reasons to focus on this attitude. First of all, it can be argued that tolerance is becoming increasingly important as a social attitude, given the structural trend toward higher levels of ethnic and cultural diversity in most Western societies (Côté \& Erickson, 2009; Harell, 2010). Ethnocentrism can be seen as running counter to the attitude of generalized trust as it expresses a strong preference for the exclusion of specific groups within the population. Ethnocentrism has strong consequences, both with regard to discriminatory behavior as with regard to an electoral preference for extreme right voting (Dejaeghere \& Hooghe, 2012). Second, we know that ethnocentrism is a very strong and stable attitude, that is at the heart of the complex of right wing authoritarian attitudes (Duckitt \& Farre, 1994). In opinion research, ethnocentrism is usually one of the strongest attitudinal scales that is also clearly onedimensional (Kinder \& Kam, 2010). The third argument is that ethnocentrism was also used as a dependent variable in the original formulation of the selection and adaption model, and in order to ensure full comparability with this first study, that was only based on a crosssectional observation, we considered it a safe option to repeat this measurement (Hooghe, 2003).

Ethnocentrism was measured using a three item scale, taken from the European Social Survey battery. Given the fact that in Western Europe ethnic diversity mainly is associated with the inflow of immigrants, this scale focuses strongly on the perception of the consequences of immigration (Sides \& Citrin, 2007). These items refer both to the perceived cultural and economic threat of increased ethnic diversity:

- According to you, is it in general good or bad for the Belgian economy that people from other countries come here? $(0=$ bad for the economy to $10=\operatorname{good}$ for the economy) 
- Do you think that the cultural life in Belgium in general is undermined or enriched by people from other countries that came to live here? $(0=$ cultural life is undermined to $10=$ cultural life is enriched)

- Has Belgium become a worse or a better place to live by the presence of people from other countries that came to live here? $(0=$ worse place to $10=$ better place $)$

These three items constitute a reliable and one-dimensional scale, both in 2006 (one factor, explained variance: $75.46 \%$; Cronbach's $\alpha$ : 0.837) and in 2008 (one factor, explained variance: $76.66 \%$; Cronbach's $\alpha$ : 0.846). In the appendix, the detailed results of the factor analyses are presented. The coding of the items was reversed, so that a higher score on the scale means a more ethnocentric attitude.

Associational Membership. In order to measure the membership of voluntary associations, respondents were presented with a list of 15 different kinds of associations. For each one of the associations, respondents could indicate whether or not they had participated in an active manner in one or more of these associations during the previous 12 months. In theory, respondents could be members of all 15 associations. The list included: sports clubs, hobby groups, youth clubs, youth associations (e.g., Scouts), cultural associations, music groups, new social movements (e.g. environmental, peace, Third World, anti-racism associations), volunteering organizations, school council, political parties, neighborhood assemblies, ethnic organizations, religious groups and the youth chapters of trade unions. These groups were chosen because earlier surveys indicate that a vast majority of youth membership in the Belgian context belongs to one of these categories of associations (Quintelier, 2008). ${ }^{2}$

Control variables. Self-evidently, various control variables had to be included: gender, education level, citizenship status, the time spent on watching television and religious participation, and again this closely mirrors the variables that were used by Hooghe (2003) and other published research on participation and ethnocentrism (Brehm \& Rahn, 1997; Côté \& Erickson, 2009; Iglič, 2010; Van der Meer \& van Ingen, 2009). Gender will be coded as a dummy where 0 indicates 'girl' and 1 'boy'. Since all the respondents in this survey are still enrolled in the school system, it is not possible yet to include information about the final education level the respondents will reach. Instead we asked respondents about their educational aspirations, by questioning them about the education level they think they will reach (high school drop-out, finishing high school, community college, university). ${ }^{3}$ The data show that adolescents are quite stable in this regard, as the educational aspirations of the respondents were measured twice, at age 16 and age 18. Correlation between both results is 
.647 ( $\mathrm{p}<0.001$ ). Citizenship status was coded as a dummy, with a score of 1 indicating that the respondent is a Belgian citizen. Additionally, we also took into account whether the respondents had one of more parents who were born outside the European Union $(=1: 0=$ both parents born in Europe). Furthermore, television time was measured by including a question on the time spent on television on an average day of the week. Answering categories ranged from 1 to 5 (never, less than 1 hour, 1 to 2 hours, 3 to 4 hours, 5 hours and more). For religious participation, too, a five point scale was used, ranging from never attending a religious service to attending more than once a week. All control variables were measured in 2006, to ascertain whether they had an effect on the dependent variable that was measured two years later. ${ }^{4}$ By measuring the independent variable two years before the dependent variable, we cannot exclude a reserve causal logic, but we do make it more unlikely that this would occur.

In the analysis, we will first ascertain whether different types of associations have an effect on ethnocentrism, controlling for prior levels of ethnocentrism, using an ordinary least squares regression. Subsequently, a structural equation model will be constructed to compare the effects of socialization and self-selection. We used the same model as Finkel $(1985 ; 2007)$. This type of cross-lagged model allows to distinguish between socialization effects and selfselection effects at the same time, and to compare these effects. On top of that, structural equation modeling allows to control for measurement error in latent variables, as this is the case for ethnocentrism.

\section{Results}

The strongest control variable is self-evidently the initial level of ethnocentrism measured during the first wave, that allows us to predict ca. thirty percent of the variance in the level of ethnocentrism two years later on. Most notably, this again supports the idea that ethnocentrism should be seen as a stable social attitude that, even at this age, does not fluctuate all that dramatically as for a substantial majority of respondents the level of ethnocentrism does not change during the observation period. Second, the educational goal of 
the respondent has a strong negative effect on the development of ethnocentrism. While in most of the earlier research education levels were shown to reduce levels of ethnocentrism, apparently questioning adolescents about their intended future education goal has more or less the same effect on attitudes. Furthermore, most of the previous studies on the occurrence of ethnocentrism are confirmed. Girls are less ethnocentric than boys, while watching television intensively has a positive effect on ethnocentrism levels. Furthermore, having at least one parent born outside the European Union is associated with lower levels of ethnocentrism, while this attitude is higher among respondents with Belgian citizenship status. This means that ethnocentrism is lower (at least taking this measurement instrument) among respondents that could be seen as a member of an ethnic minority themselves. To control for this difference, in Table 2 we also include information only for those respondents who have both parents born inside the European Union, and who thus cannot be considered as a visible minority. It can be noted that if we limit ourselves to this majority group, effects are the same. $^{5}$

Table 2. Predicting ethnocentrism for the second wave of the panel, model with control variables

\begin{tabular}{lcc}
\hline & \multicolumn{2}{c}{$\begin{array}{c}\text { Ethnocentrism 2008 } \\
\text { Only respondents with no } \\
\text { parents being born outside EU }\end{array}$} \\
\hline Ethnocentrism 2006 & $0.496^{* * * *}$ & $0.496^{* * *}$ \\
Gender $(0=$ female $)$ & $0.091^{* * *}$ & $0.096^{* * *}$ \\
Educational goal & $-0.127^{* * *}$ & $-0.136^{* * *}$ \\
Belgian citizen & $0.030^{*}$ & $0.018 \mathrm{~ns}$ \\
At least 1 parent born outside the EU & $-0.067^{* * *}$ & - \\
Attend religious services & $-0.009 \mathrm{~ns}$ & $-0.013 \mathrm{~ns}$ \\
Time spent watching television & $0.041^{* *}$ & $0.049^{* * *}$ \\
\hline $\mathrm{R}^{2}$ & 0.343 & 0.332 \\
$\mathrm{~N}$ & 3,639 & 3,399 \\
\hline $\mathrm{S}$ & & .
\end{tabular}

Source: BPPS 2006-2008. Entries are standardized effects. Control variables measured in 2006. Sign.: $\mathrm{p}<0.001: * * * ; \mathrm{P}<0.01: * * ; \mathrm{P}<0.05: *$.

These control variables, however, are not our main theoretical focus, but they allow us to construct a comprehensive model with regard to membership effects. Therefore we now list the effects of the membership variables, that each time were coded as a dummy variable (active member or not). Of the fifteen associations that were entered, only six reach levels of significance: cultural associations, youth unions, youth groups, ethnic groups, new social movements and school clubs. It has to be remembered that this was by itself already a strict test since it controlled for the initial level of ethnocentrism two years earlier. Nevertheless, 
these kind of association still manage to have a significant impact on the evolution of the level of ethnocentrism during this observation period. We do not find any effect, however, for nine other kinds of associations, and for sports clubs, political parties, religious groups and volunteering groups, the effect is almost zero (Table 3). Apparently the culture within some associations is conducive to a reduction of ethnocentrism levels, but this is clearly not the case for all associations.

Table 3. Predicting ethnocentrism for the second wave of the panel

\begin{tabular}{ll}
\hline & Ethnocentrism 2008 \\
\hline Cultural association & $-0.049^{* * *}$ \\
Youth union & $-0.040^{* *}$ \\
Youth group & $-0.040^{* *}$ \\
Ethnic group & $-0.034^{*}$ \\
New social movement & $-0.031^{*}$ \\
School club & $-0.028^{*}$ \\
Youth board & -0.026 \\
School board & -0.021 \\
Music & -0.021 \\
Youth club & -0.014 \\
Religious group & -0.009 \\
Political party & -0.007 \\
Volunteering & -0.005 \\
Sport club & 0.001 \\
Hobby group & 0.018 \\
\hline Sorce: BPS & $100-2008$. Ent
\end{tabular}

Source: BPPS 2006-2008. Entries are standardized effects obtained from 15 different regressions models, with each time the same control variables as in Table 2. Control variables measured in 2006. Sign.: p $<0.001: * * *$; $\mathrm{P}<0.01: * * ; \mathrm{P}<0.05: * . \mathrm{N}=\max 4,325$. Full regression results are reported in the appendix.

The only conclusion that can be drawn thus far is that while some associations clearly are related with lower levels of ethnocentrism, this is not the case for all kind of associations. Popular groups like sports and hobby clubs or even junior political parties clearly do not have any effect on ethnocentrism at all. The expectation that membership in general is associated with a more tolerant value pattern, therefore clearly needs to be qualified. As far as we can judge, there is just as much interaction going on in sports associations as in cultural associations, and obviously, this cannot explain the results of this analysis. However, it still can be argued that these results might be caused just as well by self-selection effects. Although it is difficult to imagine why less ethnocentric adolescents would be attracted specifically toward cultural groups or youth unions, the argument still is valid and needs to be tested.

In order to tease out the occurrence and the importance of the socialization and the selfselection effect, we conducted an additional test, that allows us to investigate whether the 
socialization effect (from participation at time-1 to ethnocentrism at time-2) is stronger than the self-selection effect (from ethnocentrism at time-1 to participation at time-2). This can be done by means of a structural equation model (Table 4). This kind of model allows us to test relations between variables like membership and attitudes that were measured during different observation time points (Finkel, 2007). Participation in voluntary associations was measured here by the membership in the six types of associations that are significantly related to ethnocentrism. The nine other types of associations were not included in the model, given the consideration that if there is no significant relation to start with, structural equation models certainly will not help us to determine the direction of that relation. In the structural equation model, we allow for both socialization and self-selection effects to occur simultaneously. 
Table 4. Relation between membership in voluntary associations and ethnocentrism

\begin{tabular}{|c|c|c|c|c|}
\hline${ }_{\text {From }}^{\text {To }}$ & Membership & $\begin{array}{c}2006 \\
\text { Ethnocentrism }\end{array}$ & $\begin{array}{c}2008 \\
\text { Membership }\end{array}$ & Ethnocentrism \\
\hline \multicolumn{5}{|l|}{2006} \\
\hline Membership & & $\begin{array}{l}-0.141(0.022) \\
-0.114 * * *\end{array}$ & $\begin{array}{c}0.540(0.017) \\
0.564 * * *\end{array}$ & $\begin{array}{c}-0.152(0.032) \\
-0.064 * * *\end{array}$ \\
\hline Ethnocentrism & & & $\begin{array}{c}-0.018(0.006) \\
-0.043 * *\end{array}$ & $\begin{array}{c}0.635(0.020) \\
0.618 * * *\end{array}$ \\
\hline \multicolumn{5}{|l|}{2008} \\
\hline Membership & & & & $\begin{array}{c}-0.058(0.016) \\
-0.070 * * *\end{array}$ \\
\hline \multicolumn{5}{|l|}{ Control variables } \\
\hline Gender $(0=$ female $)$ & $\begin{array}{c}-0.240(0.024) \\
-0.155^{* * *}\end{array}$ & $\begin{array}{c}0.476(0.061) \\
0.134 * * *\end{array}$ & & $\begin{array}{c}0.235(0.054) \\
0.064 * * *\end{array}$ \\
\hline Educational goal & $\begin{array}{c}0.146(0.015) \\
0.145^{* * *}\end{array}$ & $\begin{array}{c}-0.363(0.042) \\
-0.157 * * *\end{array}$ & & $\begin{array}{c}-0.249(0.039) \\
-0.105^{* * *}\end{array}$ \\
\hline Belgian citizen & $\begin{array}{c}0.208(0.048) \\
0.055^{* * *}\end{array}$ & $\begin{array}{c}0.907(0.144) \\
0.105^{* * *}\end{array}$ & & \\
\hline $\begin{array}{l}\text { Parent born outside } \\
\text { Europe }\end{array}$ & $\begin{array}{c}-0.300(0.042) \\
-0.101 * * *\end{array}$ & $\begin{array}{c}-1.328(0.127) \\
-0.194 * * *\end{array}$ & & \\
\hline $\begin{array}{l}\text { Time spent watching } \\
\text { television }\end{array}$ & $\begin{array}{c}0.099(0.015) \\
0.103^{* * *}\end{array}$ & $\begin{array}{c}-0.186(0.039) \\
-0.085^{* * *}\end{array}$ & & \\
\hline $\begin{array}{l}\text { Attend religious } \\
\text { services }\end{array}$ & $\begin{array}{c}-0.066(0.014) \\
-0.078 * * * \\
\end{array}$ & $\begin{array}{c}0.085(0.034) \\
0.043^{*} \\
\end{array}$ & & \\
\hline Explained variance & 0.084 & 0.132 & 0.327 & 0.460 \\
\hline
\end{tabular}

The results show first of all that both in 2006 and in 2008 membership is negatively and significantly related to ethnocentrism. This effect is somewhat stronger in $2006\left(-.114^{* * *}\right)$ than it is in $2008(-.070 * * *)$. By itself, this is just a cross-sectional finding that confirms the result of earlier research. Second it can be observed that membership in 2006 is strongly related to membership in $2008(.564 * * *)$. This again means that already at this age, participatory patterns clearly are not transient, and respondents who were active at age 16 also tend to remain active at age 18. The same kind of relation can be observed for ethnocentrism. Here we find a relation of $.618^{* * *}$, indicating that ethnocentrism is a stable attitude that is rather resistant to sudden changes.

Finally we arrive at our crucial test, where we use membership during wave 1 to explain ethnocentrism at wave 2 . This socialization effect is clearly significant $(-.064 * * *)$. The opposite effect, however, is just as clearly significant $\left(-.043^{* * *}\right)$ which implies that those who 
are less ethnocentric during wave 1, are far more likely to join these associations at wave 2 . The conclusion to be drawn from these results, therefore is that both self-selection and socialization effects occur. In this regard, our longitudinal design confirms earlier findings by Brehm and Rahn (1997) showing that both effects can be observed.

However, the question still remains whether the differences between both effects is significant. The current analysis suggests that the socialization effects tend to be stronger than the self-selection effects. If we run an additional test by constraining both effects to be equal, we can observe that the socialization effect is indeed significantly stronger than the selfselection effect $\left(\mathrm{Chi}^{2} \Delta: 18.648^{* * *}, 1 \mathrm{df}\right)$. Therefore, we can safely conclude that while both socialization and self-selection effects are present, the socialization effect, at least among this age group, is clearly present and is significantly stronger than the self-selection effect. As predicted by the selection and adaption model, socialization effects furthermore do not occur in a uniform manner across groups, but only in specific forms of associations. The current analysis does not allow us to determine in an exact manner what are the defining characteristics of those associations that are associated with a reduction of ethnocentrism. The data show that the ethnic diversity of these associations is not fundamentally different from that of other associations. It can be assumed however, that especially in cultural associations, youth unions and other groups, a more tolerant group culture is present. More in-depth research, however, is necessary to ascertain whether this in a reliable manner could be invoked as a causal mechanism.

\section{Discussion}

Already since Tocqueville's classical writings from the early 19th century, it has been repeated again and again that membership in voluntary associations has a strong socialization effect on the members of these associations. Convincing empirical evidence for this bold claim, however, has been rather scarce, as it was mostly based on cross-sectional data. This kind of data, obviously do not allow us to determine the direction of causality between both phenomena. In this article we used two year panel data from a representative sample of Belgian late adolescents, which should provide us with more insights on possible causal mechanisms. The results first of all confirm that there certainly is not an automatic relation between any kind of membership and a reduction of ethnocentrism. As was already observed in the introduction: there is indeed no causal mechanism available that could explain such an effect. The most obvious conclusion to be drawn from this observation is that voluntary 
associations do not offer a panacea solution with regard to the development of democratic attitudes. It is important to note, furthermore, that sports associations most likely are among the most diverse associations for Belgian adolescents, as teenagers from an immigrant background also find their way to these kind of associations. This level of ethnic diversity within the association, however, does not seem to have an effect of ethnocentrism levels.

For other kinds of associations, however, the assumptions were met. Membership of cultural associations, ethnic groups, youth unions and youth clubs is significantly associated with reduced levels of ethnocentrism, even controlling for the initial level of ethnocentrism, which in most cases is already lower among members of this kind of associations. ${ }^{6}$ It can only be assumed, therefore that elements of the culture that is prevalent in these associations, or the interaction patterns in the association are associated with a reduction in ethnocentrism. It falls outside the scope of the current analysis to investigate what element of the functioning of these groups exactly is responsible for this effect. Furthermore it has to be remembered that this study does not allow for conclusions about specific associations, but only about groups of associations that fit together into one category. If we further want to investigate whether indeed specific group cultures are responsible for the observed effects, this line of study should be pursued with in-depth observations within specific associations. In any case, however, the current results confirm the hypothesis that only specific associations with have this kind of effect. The assumption that socialization effects of participation are group-specific also allows us to arrive at a better understanding of what has been called the dark side of social capital. It has been suggested that non-democratic associations might have a detrimental effect on pro-social values, but thus far this claim was not based on a viable causal mechanism (Mondak \& Sanders, 2005). The current study suggests that if, within an association, non-democratic attitudes are prevalent and receive support, this most likely will strengthen these attitudes, thus effectively contributing to a 'dark side' of social capital. This implies that the potential impact of voluntary associations also needs to be qualified. As Turner et al. (1987) already suggested: the socialization effect of voluntary associations is not able to alter in a fundamental manner pre-existing value patterns. The current analysis suggest that ethnocentrism levels of members are already lower at the moment they enter, and that subsequently they continue to decline. Dropping a highly intolerant teenager into a tolerant association would be interesting as a social experiment, but most likely it would not lead to the socialization effect that we have described, as that is dependent on the fact there is already a match between the value pattern of the new member and the association that $\mathrm{s} / \mathrm{he}$ joins. 
Other caveats, too, are in order. First of all it has to be remembered that this study was conducted among adolescents, and most likely their value pattern is more open to change than the attitudes among adults. Nevertheless, when comparing their answers during the first and the second wave of the study, it could be observed that both their participatory behavior as their level of ethnocentrism was rather stable. Second, the observation period was limited to two years, so it remains to be investigated whether longer periods of membership and activity within the associations are associated with the cultivation of matching value patterns among the members. Third, furthermore, data remained limited to Belgium, so we cannot claim that these findings could be generalized toward other cultural contexts. There is no apparent reason to assume however, that the Belgian context would be highly exceptional compared to other countries in Western Europe. Fourth, while the longitudinal design of our study has obvious advantages in comparison to a cross-sectional analysis, we have to acknowledge that this design too does not allow us any final conclusions with regard to causality. It has to be remembered that all of the respondents in the sample grew two years older during the research project, and this might lead to maturation effects that we cannot fully control for. Even in that case, however, we need to explain why maturation effects would be different among members of some associations compared to other respondents.

We can claim therefore that this longitudinal study provides new arguments for the validity of the hypothesis about the occurrence of a socialization effect. This by no means implies that self-selection should be absent, as the structural equation model reported in this article demonstrates in a convincing manner that self-selection is clearly present. Socialization effects, however, proved to be significantly stronger than self-selection effects, thereby confirming the importance of membership of voluntary association in shaping tolerant and democratic attitudes.

In line with the selection and adaption perspective, it must be emphasized that not all kinds of associations have this effect. Apparently this effect is only present if the culture that is present within an association also is conducive to reducing prejudice. This is in line with the insights gathered from group psychology, showing that members tend to adapt to the specific value patterns that receive authoritative support within an interaction context. As far as we know, this is indeed the first time that longitudinal evidence is presented in favour of this perspective, demonstrating that groups exert specific socialization effects even controlling for initial levels of attitudes. These findings do not necessarily invalidate the Tocquevillian optimism about the social role of voluntary associations, as it can be assumed that associations where distrust, ethnocentrism or cynicism are being cultivated, only form a very 
small part of the total number of associations within a society. The most obvious conclusion to be drawn from these results is therefore that associational membership does remain important as a resource for democratic societies. However, not any kind of association will have the assumed effect. Only if a specific value pattern is upheld and receives support within the association, it can also be expected that membership of this association will lead to a further reduction of prejudice.

\footnotetext{
1 . On average, respondents that were reached again in the same school, were more active and more tolerant than those who responded by mail. This does not necessarily point to method effects, however, since the adolescents that were contacted at school again do have different background characteristics (they did not switch schools, they were present at the school, and the progressed in a normal manner from the $4^{\text {th }}$ to the $6^{\text {th }}$ grade of high school $\left(10^{\text {th }}\right.$ and $12^{\text {th }}$ grade in the US system) in two years time).

${ }^{2}$. In an additional analysis, we also allowed for multiple memberships but this did not lead to different results.

${ }^{3}$. This measurement also taps the socio-economic status of the respondent. The advantage of this proxy-variable is that it has very few missing cases, which is not the case for more obvious operationalizations like the education level of the mother and/or the father. An alternative analysis using this indicator of socio-economic status led to similar but weaker effects.

4. Additional controls were implemented for school type and language (French or Dutch) of the respondent, but these did not lead to significant results (results available from the authors). 5 . Other operationalizations of 'ethnic minority group' were tried too, but the strongest delineation of this group proved to be to have both parents being born inside the European Union.

${ }^{6}$. Especially the membership of an 'ethnic group' might seem counterintuitive in this regard. It has to be noted, however, that this effect remains robust even among those respondents where both parents were born in Belgium. In practice, therefore, this groups refers to associations that are focused on non-European cultures etc., and they do not refer to any effort to strengthen or confirm ethnic identity of the members.
} 